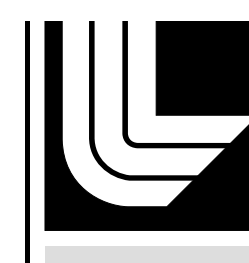

LAWRENCE LIVERMORE N A T IO N A L LABORATORY

\title{
Surrogate Nuclear Reactions and the Origin of the Heavy Elements
}

J. Escher, L. Ahle, L. Bernstein, J.A. Church, F. Dietrich, C. Forssen, R. Hoffman

July 19, 2004

The Eight International Symposium on Nuclei in the Cosmos Vancouver, Canada July 19, 2004 through July 23, 2004 
This document was prepared as an account of work sponsored by an agency of the United States Government. Neither the United States Government nor the University of California nor any of their employees, makes any warranty, express or implied, or assumes any legal liability or responsibility for the accuracy, completeness, or usefulness of any information, apparatus, product, or process disclosed, or represents that its use would not infringe privately owned rights. Reference herein to any specific commercial product, process, or service by trade name, trademark, manufacturer, or otherwise, does not necessarily constitute or imply its endorsement, recommendation, or favoring by the United States Government or the University of California. The views and opinions of authors expressed herein do not necessarily state or reflect those of the United States Government or the University of California, and shall not be used for advertising or product endorsement purposes. 


\section{Surrogate Nuclear Reactions and the origin of the heavy elements}

J. Escher, L. Ahle, L. Bernstein, J.A. Church, F. Dietrich, C. Forssén, and R. Hoffman ${ }^{\text {a* }}$

${ }^{a}$ Lawrence Livermore National Laboratory, P.O. Box 808, Livermore, CA 94551, U.S.A.

An innovative method for indirectly determining reaction cross sections via Surrogate Nuclear Reactions is presented. Exploring indirect approaches for obtaining reaction cross sections is important since a large number of nuclear reactions relevant to astrophysics cannot be measured with currently available techniques. A program is outlined for developing a comprehensive framework for planing and interpreting experiments that can yield the cross sections of interest. The applications will focus on reactions involving unstable nuclei that play a key role in the production of the elements between iron and uranium.

\section{THE SURROGATE APPROACH}

Determining cross sections for reactions on short-lived nuclear species is a major challenge for nuclear physics and nuclear astrophysics. Many of these nuclei are too difficult to produce with currently available experimental techniques or too short-lived to serve as targets in present-day set-ups. Some nuclear reactions will remain unmeasurable even at upcoming and planned radioactive-beam facilities. It is therefore important to explore alternative methods for determining reaction cross sections involving unstable nuclei.

The Surrogate Nuclear Reaction technique can be employed to determine in-

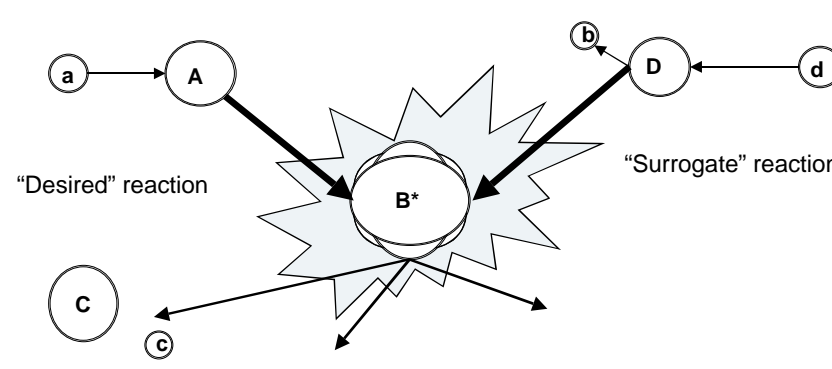

Figure 1. Schematic representation of the Surrogate reaction mechanism.

directly the cross section for a particular type of "desired" reaction, namely a two-step reaction, $a+A \rightarrow B^{*} \rightarrow c+C$, that proceeds through a compound nuclear state $B^{*}$, see Figure 1 . The Surrogate idea is based on the assumption that the formation and decay of a compound nucleus $(\mathrm{CN})$ are independent of each other (for each angular momentum and parity value). In such situations, the

cross section for the "desired" reaction can be (somewhat schematically) expressed as

$\sigma_{\alpha \chi}=\sum_{J, \pi} \sigma_{\alpha \chi}(E, J, \pi) \quad$ with $\quad \sigma_{\alpha \chi}(E, J, \pi)=\sigma_{\alpha}^{C N}(E, J, \pi) G_{\chi}^{C N}(E, J, \pi)$.

*This work was performed under the auspices of the U.S. Department of Energy by the University of California, Lawrence Livermore National Laboratory (LLNL) under contract No. W-7405-Eng-48. The project (04-ERD-057) is funded by the Laboratory Directed Research and Development Program at LLNL. 
Here $\alpha$ denotes the entrance channel $a+A$ and $\chi$ represents the relevant exit channel $c+C$. In many cases the formation cross section $\sigma_{\alpha}^{C N}=\sigma\left(a+A \rightarrow B^{*}\right)$ can be calculated reasonably well by using optical potentials, while the theoretical decay probabilities $G_{\chi}^{C N}$ for the different channels $\chi$ are often quite uncertain. The objective of the Surrogate method is to determine these decay probabilities experimentally.

In the Surrogate approach, the compound nucleus $B^{*}$ is produced via an alternative ("Surrogate"), direct reaction $d+D \rightarrow b+B^{*}$ and the decay of $B^{*}$ is observed in coincidence with the outgoing particle $b$. The probability for forming $B^{*}$ in the Surrogate reaction (with specific values for the excitation energy $E$, angular momentum $J$, and parity $\pi)$ is $F_{\delta}^{C N}(E, J, \pi)$, where $\delta$ refers to the entrance channel $d+D$. The quantity

$P_{\chi}(E)=\sum_{J, \pi} F_{\delta}^{C N}(E, J, \pi) G_{\chi}^{C N}(E, J, \pi)$

which gives the probability that the compound nucleus $B^{*}$ was formed with energy $E$ and decayed into channel $\chi$, can be obtained experimentally. The direct-reaction probabilities $F_{\delta}^{C N}(E, J, \pi)$ have to be determined theoretically, so that the decay probabilites $G_{\chi}^{C N}(E, J, \pi)$ can be extracted from the measurements and, subsequently, inserted in Eq. (1) to yield the desired reaction cross section. The Surrogate technique is particularly valuable when the target of interest, $A$, is short-lived and a suitable Surrogate reaction involving a stable target $D$ can be identified.

\subsection{Early Surrogate Experiments}

The Surrogate approach was used in the 1970s, albeit in a very simplistic manner, to extract $(n, f)$ cross sections from $t$-induced or ${ }^{3}$ He-induced transfer reactions [1]. Measured fission probabilities, $P_{f}$, were simply multiplied by an estimated cross section for the formation of the compound nucleus in the neutron-indued reaction of interest: $\sigma_{(n, f)} \approx \sigma_{n}^{C N} P_{f}$. While the resulting $(n, f)$ cross section estimates agreed with direct measurements (where available) to about 10-20\% for incident neutron energies above about $1 \mathrm{MeV}$, it resulted in serious discrepancies below $1 \mathrm{MeV}$, which were attributed to a) large uncertainties in the low-energy optical-model calculations employed, and b) the neglect of the difference in the angular-momentum populations of the compound nucleus in the Surrogate (direct) and "desired" (neutron-induced) reactions (see " $J$ population mismatch" in Section 2).

In addition to fission measurements, some early experiments were carried out to assess the feasibility of using the Surrogate technique to determine cross sections for $(n, \alpha)$ and $(n, p)$ reactions on nuclei in the mass-90 region [2]. These experiments highlighted further issues that needed to be addressed in order to extract reliable cross sections from Surrogate measurements. In particular, the effects of projectile break-up in the Surrogate reaction needed to be estimated and ambiguities in identifying the reaction sequence in some reactions needed to be resolved. It was concluded that a comprehensive theory effort would be required to eliminate these uncertainties.

\subsection{Revisiting the Surrogate Method}

Recently, Younes and Britt [3] revisited the Surrogate $(t, p f)$ and $\left({ }^{3} \mathrm{He}, x f\right)$ fissioncorrelation measurements from the 1970s. They employed a simple direct-reaction model to compensate for the angular-momentum population difference between neutron-induced 
and $(t, p)$ reactions and used improved optical-model calculations to obtain $(n, f)$ cross sections for various $\mathrm{Pu}, \mathrm{U}$, and Th isotopes. For the benchmark case ${ }^{235} \mathrm{U}$, they obtained significant improvements over the early Surrogate work: For neutron energies above about $0.5 \mathrm{MeV}$, the extracted cross sections showed agreement to within $10 \%$ with the accepted values in the evaluated nuclear data file (ENDF), and for energies between $0.1 \mathrm{MeV}$ and $0.5 \mathrm{MeV}$, the difference was at most $20 \%$. In addition to reproducing the fission cross section for the $J^{\pi}=7 / 2^{-}$ground state of ${ }^{235} \mathrm{U}$, Younes and Britt were able to estimate the fission cross section for the isomeric $1 / 2^{+}$state at $77 \mathrm{eV}$, which to date has not been measured directly. Their results are in general agreement with calculations by Lynn and Hayes [4] and illustrate the promise of the Surrogate technique.

\section{DEVELOPING THE SURROGATE TECHNIQUE}

Before the Surrogate approach can be employed to obtain cross sections for reactions involving a wide range of unstable nuclei, it is necessary to a) develop a framework for planning and interpreting Surrogate experiments, b) establish the validity of the method for each region of interest, and c) determine the limitations of the technique. A new research program at Lawrence Livermore National Laboratory (LLNL) aims at addressing these issues. The strategy of the program is to combine the development of theoretical tools and models with experiments suitable for testing the theory.

In order to develop a framework for planing and interpreting Surrogate experiments, the following challenges need to be addressed: 1 . The " $J^{\pi}$ population mismatch". In the early work it was assumed that the compound nuclear state that is prepared in the Surrogate reaction is similar to the state formed in the original, "desired" reaction. In most cases, however, the Surrogate reaction populates the states in the intermediate nucleus differently than the desired $a+A$ channel. In particular, the angular-momentum and parity $\left(J^{\pi}\right)$ populations will be "mismatched". Since the $J^{\pi}$ population influences the decay probabilities of the compound nucleus, models have to be developed which account for the differences between the reactions. More details are given in Ref. [5]. 2. Identification of the final reaction product(s). To obtain the experimental decay probabilities $P_{\chi}(E)$ for a particular exit channel $\chi=C+c$, it is essential to identify the reaction product(s) in that channel. For some important applications of the Surrogate technique, this identification involves measuring characteristic $\gamma$ rays from the de-excitation of the desired reaction product $C$. In order to convert the measured $\gamma$-ray intensities to channel probabilities $P_{\chi}(E)$, a model for the de-excitation process is needed, which, in turn, requires a proper description of structural properties of the remnant $C$, such as level densities, branching ratios between discrete levels, and internal conversion rates.

To establish the validity of the Surrogate approach, it is necessary to carry out experiments that provide benchmarks. For example, the reaction ${ }^{92} \operatorname{Zr}\left(\alpha, \alpha^{\prime}\right)^{92} \mathrm{Zr}^{*}$ can be used as a Surrogate for $n+{ }^{91} \mathrm{Zr} \rightarrow{ }^{92} \mathrm{Zr}^{*}$. Observing the decay ${ }^{92} \mathrm{Zr}^{*} \rightarrow{ }^{92} \mathrm{Zr}$, by $\gamma$-ray emission, in coincidence with the outgoing $\alpha$ particle allows one to infer the ${ }^{91} \mathrm{Zr}(n, \gamma)^{92} \mathrm{Zr}$ cross sections, which can then be compared to direct measurements. Reference [6] describes the details of a Surrogate benchmark experiment.

A comprehensive study of the Surrogate approach must include a careful investigation of the limitations of the method. In particular, the following issues need to be consid- 
ered: 1. The role of pre-equilibrium reactions. Central to the Surrogate method is the assumption that the formation and decay of the intermediate nuclear state - in both the "desired" and the Surrogate reaction - are independent of each other (apart from conserving constants of motion). This is only valid if the intermediate nucleus equilibrates (becomes a "compound" nucleus) before it decays into the final reaction products. Rapid ("pre-equilibrium") decay of the intermediate configuration before a compound nucleus can be formed would invalidate the Surrogate analysis. The probability that a compound nucleus is actually formed in a particular reaction needs to be estimated and the effects of pre-equilibrium decays on energy balances and $J^{\pi}$ populations have to be studied. 2 . The role of projectile break-up. If a stripping reaction such as $(d, p)$ or $\left({ }^{3} \mathrm{He}, d\right)$ is to be used in the Surrogate experiment, projectile break-up in the Coulomb-plus-nuclear field of the target nucleus may produce an outgoing particle which can be incorrectly interpreted as resulting from a transfer reaction. It is important to estimate the relevant break-up probabilities and, if necessary, employ experimental techniques that minimize this problem.

\section{APPLICATIONS OF THE METHOD}

While the Surrogate method can (in principle) be used to determine cross sections for reactions on a large variety of nuclei, its greatest value lies in applications that involve unstable isotopes. Cross sections for unstable nuclei are not only very difficult to measure, they also play an important role for addressing some of the most compelling questions of basic science. Among the unanswered mysteries about the nature and evolution of our universe is the origin of the heavy elements [7]. Much effort is currently being devoted to exploring nuclear processes (such as the s and r processes) and astrophysical environments that can produce the elements between iron and uranium. Of particular interest in the context of the s process are branch points, unstable nuclei that are produced in the $\mathrm{s}$ process with a life time long enough to allow the s process to proceed by either neutron capture or $\beta$ decay. A very important ingredient for determining the probability of one path dominating over the other is the associated neutron-capture cross section. Determining neutron-capture cross sections on s-process branch points is an important application of the Surrogate method.

\section{REFERENCES}

1. J.D. Cramer and H.C. Britt, Nucl. Sci. and Eng. 41, 177 (1970); H.C. Britt and J.B. Wilhelmy, ibid. 72, 222 (1979). B. B. Back et al., Phys. Rev. C 10, 1948 (1974); ibid. 9, 1924 (1974); J. D. Cramer and H. C. Britt, ibid. 2, 2350 (1970).

2. H. C. Britt and J. B. Wilhelmy, private communication.

3. W. Younes and H.C. Britt, Phys. Rev. C 67, 024610 (2003); ibid. 68, 034610 (2003).

4. J.E. Lynn and A.C. Hayes, Phys. Rev. C 67, 014607 (2003).

5. C. Forssén et al., 8th Internat. Symposium on Nuclei in the Cosmos, Vancouver, 2004.

6. J.A. Church et al., ibid.

7. Board on Physics and Astronomy, National Research Council, Connecting Quarks to the Cosmos: Eleven Science Questions for the New Century, (National Academies Press, Washington, D.C., 2003). 